\title{
BMJ Open Moving 2 Mindful (M2M) study protocol: testing a mindfulness group plus ecological momentary intervention to decrease stress and anxiety in adolescents from high-conflict homes with a mixed-method longitudinal design
}

\author{
Rachel Lucas-Thompson (10 , ${ }^{1,2}$ Natasha Seiter, ${ }^{1}$ Patricia C Broderick, ${ }^{3}$ \\ James Douglas Coatsworth, ${ }^{1}$ Kimberly L Henry, ${ }^{2}$ Charlotte J McKernan, ${ }^{1}$ \\ Joshua M Smyth ${ }^{3}$
}

To cite: Lucas-Thompson $\mathrm{R}$, Seiter N, Broderick PC, et al. Moving 2 Mindful (M2M) study protocol: testing a mindfulness group plus ecological momentary intervention to decrease stress and anxiety in adolescents from high-conflict homes with a mixed-method longitudinal design. BMJ Open 2019;9:e030948. doi:10.1136/ bmjopen-2019-030948

- Prepublication history for this paper is available online To view these files, please visit the journal online (http://dx.doi org/10.1136/bmjopen-2019030948).

Received 09 May 2019 Revised 06 November 2019 Accepted 07 November 2019

D Check for updates

(c) Author(s) (or their employer(s)) 2019. Re-use permitted under CC BY-NC. No commercial re-use. See rights and permissions. Published by BMJ.

For numbered affiliations see end of article.

Correspondence to Dr Rachel Lucas-Thompson; Lucas-thompson.rachel. graham@colostate.edu

\section{ABSTRACT}

Introduction Interparental conflict exposure places adolescents at risk for problems with stress and anxiety; existing prevention/intervention strategies focus on reducing interparental conflict. Mindfulness-based programmes may be a promising treatment strategy for this population, but studies have not yet tested whether they are effective in this high-conflict context. In addition, evidence suggests that extensions to traditional treatments, such as delivering components in daily life that are tailored to moments of need can increase treatment efficacy, particularly when combined with in-person treatments and particularly for adolescents. However, there are no such extensions to mindfulness interventions available. The Moving 2 Mindful study aims to (1) develop an ecological momentary intervention (EMI) supplement to Learning to BREATHE (L2B), an evidencebased mindfulness intervention for adolescents; (2) refine the EMI programme and determine the best delivery plan; (3) examine the feasibility and acceptability of L2B Plus (L2B plus the developed supplement) and (4) examine the potential for $L 2 B$ Plus to reduce stress and anxiety for adolescents from high-conflict homes.

Methods and analysis The Moving 2 Mindful study proposes a mixed-methods approach to developing and refining a multimethod adaptive supplement to L2B. Feasibility, acceptability and potential effectiveness will be tested in a sample of 38 families, who will be randomly assigned to receive $\mathrm{L} 2 \mathrm{~B}$ Plus or an active health and wellness control condition and followed until 3 months postintervention. A range of psychosocial and physiological factors will be assessed at multiple time points. This study is registered with clinicaltrials.gov (ID NCT03869749; preresults).

Ethics and dissemination The Institutional Review Board at Colorado State University has approved this study. Findings will be disseminated in scientific journals and conferences, whether they are positive, negative or inconclusive.
Strengths and limitations of this study

A strength of the current study is the use of a randomised controlled trial design.

- One of the main strengths of the design is the mixed method longitudinal assessment.

- A limitation is a sample size that is relatively small (although in line with goals of documenting feasibility and acceptability), which limits the generalisability of findings and reduces statistical power.

\section{INTRODUCTION}

Adolescence is a unique and critical period for the development or prevention of stressregulatory problems that have life-span consequences. ${ }^{1}$ Therefore, initiatives to promote positive coping among vulnerable adolescents are critical. Children and adolescents who witness hostile, frequent and poorly resolved interparental conflict are at increased risk for problems with stress regulation as well as anxiety (see figure 1). ${ }^{2-6}$ Although these youth often experience stress and anxiety symptoms above clinical thresholds, ${ }^{7}$ even problems that are below clinical thresholds can have long-term adverse consequences. ${ }^{8-10}$ Interparental conflict is to some extent normative and inevitable; most couples experience some constructive as well as destructive marital conflict. ${ }^{11}$ In addition, the hostile and frequent conflict that is the most damaging for children and adolescents is relatively prevalent in the USA. ${ }^{12}$ The purpose of the current study is to investigate the efficacy 


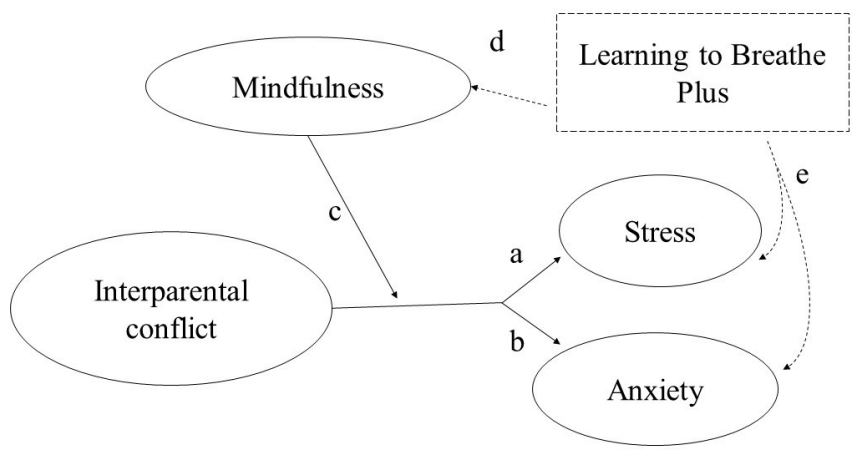

Figure 1 Conceptual model guiding this study. Past work provides evidence for paths ' $a$ ' and ' $b$ '. After developing and refining Learning to Breathe Plus (Aims \#1 and \#2) and evaluating its feasibility and acceptability (Aim \#3), we will test hypothesised path ' $c$ ' and explore whether there is experimental support for paths ' $d$ ' and 'e' (Aim \#4).

of a mindfulness intervention for reducing stress and anxiety for adolescents from high-conflict homes and also to develop, refine and test feasibility/acceptability of a multimethod adaptive intervention supplement to this mindfulness intervention.

Given the consequences of exposure to interparental conflict for adolescents, it is important that adolescents from high-conflict homes be targeted in prevention of the development of long-term problems with stress and anxiety. There are, however, very few existing programmes that directly serve them. Reducing destructive communication between marital partners has been a target of most of these programmes. ${ }^{13-15}$ Some evidence suggests that improving marital interaction is beneficial to children. ${ }^{1416}$ Not all studies that investigate the effect of marital interventions on children suggest significant benefits and effect sizes have typically been small (ie, $d<0.10) .{ }^{16}$ It may also be insufficient to focus exclusively on improving marital relationships to address adolescent problems. An approach that neglects to target youth specifically, in lieu of targeting marital conflicts that affect them, may overlook the possible impact of a more direct approach. In high-conflict marital relationships, efforts to change these interaction patterns are often challenging, time-consuming and costly. ${ }^{15}$ For example, partners may not be willing or committed to the intensive work needed to change habitual interaction patterns. Given that their parents' commitment to change is out of adolescents' control, focusing intervention efforts exclusively on parents takes agency out of the hands of adolescents. Interventions that directly target adolescents from high-conflict homes and aim to improve their emotion regulation present a potential alternative way of reducing anxiety and stress in this population. However, to our knowledge, there has been no research investigating the efficacy of such interventions.

Research suggests that interventions that increase mindfulness (ie, present-focused, non-judgmental awareness) are effective at reducing problems with stress and anxiety. ${ }^{17}$ These approaches are also accepted by, and effective for, adolescents. ${ }^{18}$ Mindfulness interventions directly targeting adolescents do not require that parents be committed to change. One particular programme, Learning to BREATHE (L2B), is an evidence-based programme that is well-liked by adolescents (eg, Broderick and Metz, 2009). Activities include psychoeducation about stress and emotion regulation and practices of body scanning, non-aerobic yoga and meditation, which are designed to cultivate and provide opportunities to practice present-focused, non-judgmental attention. In addition, this programme can be implemented in schools or in the community, in varying numbers of sessions and session lengths. In addition to increasing mindfulness, L2B directly targets the regulatory problems that are often displayed by adolescents from high-conflict homes. ${ }^{19-26}$ Therefore, L2B may be an effective intervention strategy to use to improve functioning in adolescents from high-conflict homes. ${ }^{27}$

Interventions that incorporate extensions to traditional treatments such as delivering intervention components in daily life that are tailored to moments of need (ecological momentary intervention (EMI) strategies; eg, text-messaged intervention content) often increase the efficacy of treatments. ${ }^{28} 29$ EMI strategies have been shown to have medium-sized effects on a variety of mental health outcomes including anxiety, depression, perceived stress, acceptance, relaxation and quality of life. They are effective as both primarily stand-alone interventions ${ }^{30}$ and especially as supplements to in-person interventions. ${ }^{31}$ In addition, EMIs are particularly well-liked by adolescents. ${ }^{28}$ Cell-phone use is integral to the lives of adolescents today. According to a 2018 survey, $95 \%$ of teens have or have access to a smart phone. ${ }^{32}$ Adolescents endorse texting as their preferred method of communication and are much more likely to communicate via text messages rather than face-to-face, especially out of school; in fact, the median number of text messages sent by teens per day is $60 .{ }^{33} 34$ Given the frequency of their cell-phone use, EMIs may provide a natural and effective way to provide intervention content to adolescents. Despite the power of these EMI supplements, as far as we are aware, no empirically supported EMIs are available for mindfulness-based group interventions. As such, it is important to develop an evidence-based and empirically supported EMI supplement to mindfulness-based group interventions to maximise potential to support long-term positive change for adolescents. $^{35}$

\section{Aims}

The aims of this study are to (1) develop an EMI supplement to L2B; (2) refine the EMI supplement and determine the best delivery plan; (3) examine the feasibility and acceptability of L2B Plus (L2B plus the developed supplement) and (4) examine the extent to which L2B Plus reduces stress and anxiety for adolescents from high-conflict homes. We hypothesise that L2B Plus will decrease stress and anxiety relative to an active control condition. 


\section{METHODS AND ANALYSIS}

\section{Patient and public involvement}

Patients/members of the public informed the development/refinement of the EMI supplement to the programme as described below, but otherwise patients/ members of the public were not involved in other elements of study design or implementation. Also detailed below is information about how participants will assess the burden of the intervention via reports of acceptability. Participants will have the option to have aggregated results disseminated to them via mail or email after study completion; results will also be available for participants on a study website.

\section{Aim 1: Develop a multimethod adaptive intervention supplement to L2B \\ Overview}

There will be two elements to the supplement to L2B: (1) EMI in the form of text messages that provide participants with (i) reminders to practice mindfulness, (ii) reminders about types of mindfulness practices to engage in (linked to the content from the last group meeting) and (iii) encouragements to incorporate mindfulness into daily life and, (2) an on-demand library of mindfulness education and practices (including audios, videos and written articles with images), which adolescents will always have access to, and will be directed to when they report (via ecological momentary assessments (EMA)) feeling stressed, feeling anxious or exposed to family conflict.

To create the intervention text-messages, 30 adolescents (in cohorts of 4-10 members) will experience the full L2B programme and engage in an activity designed to elicit suggestions from programme participants about potential text-messages. Thirty adolescents will be recruited to participate in L2B to assist with facilitator training/ certification and will also be asked to contribute to textmessage development in this way. More specifically, at the end of each session, each adolescent will be asked to write down one or two sentences that could serve as reminders and encouragements to practice. These will serve as inspiration for some of the text-messages sent to participants; the study team will also create such reminders and encouragements to be sent via text message. The study team will review this pool of potential messages to select those that are most in line with the goals of each session goals. In addition, very short practices will be included in the text message component. This development process is currently ongoing.

The research team has already completed the initial development of the on-demand library of mindfulness education and practices. Two strategies were used to create this on-demand library. First, the research team conducted a thorough search of available online mindfulness education and practices. Second, the research team identified gaps in this existing content and created new content to fill those gaps. In evaluating and collating the content for the library, the following exclusion criteria were applied: (1) technical problems (eg, low sound quality); (2) free use prohibited; (3) specific/explicit religious references in language or image; (4) developmentally inappropriate content (eg, language inconsistent with the experience of youth and/or young child or adult focused); (5) length $>20 \mathrm{~min}$; (6) unclear/confusing guidance for practice (eg, contradictory instructions); (7) pedagogical inappropriateness (ie, not reflective of programme themes or practices taught in group) and, finally, (8) types of mental training (ie, mantra meditation, visualisation) that may be well-presented but which are not included in L2B.

Regarding the eighth criteria mentioned above, the family of 'contemplative, meditative practices' represent a range of different forms of mental training. L2B is derived from the same mental training/meditative tradition used in mindfulness-based stress reduction (MBSR). This tradition defines mindfulness as a particular form of attention that is purposeful, present-focused and nonreactive. Mindfulness many be practiced by establishing stability of attention (focus) and by cultivating awareness of whatever is occurring (open awareness) with the attitude of non-judgment, curiosity and acceptance of experience. Three families of practice are taught in MBSR: focused attention, open awareness and compassion practice. These are distinct from other types of contemplative practices such as mantra meditation, analytical meditation, visualisation, relaxation training and so on. Although these are all important forms of mental training (and share similar goals), they are not emphasised in L2B. Independent practices that train attention in ways consistent with L2B practices were selected to avoid confusion for participants and to reinforce what is being taught in the group programme.

We sought content within each specific topic that varied in length (specifically, categories of $0-5 \mathrm{~min}, 6-10 \mathrm{~min}$ and $11+\mathrm{min})$. Finally, all included content was sorted to determine the L2B 'theme' to which it applied. The basic principle guiding this sorting was to provide opportunities for students to practice independently those skills that have already been introduced and practiced in class. The practices in L2B are scaffolded sequentially; practices were assigned to themes in accordance with this progression. Care also was taken to select materials that would match students' level of understanding and ability. For example, in early sessions, practices focus more narrowly on one object of focused attention (eg, breathing); in later sessions, practices increase in complexity as participants are guided to focus on multiple objects of attention (eg, body, thoughts and feelings). Practices in the on-demand library were sorted according to the same progression. Second, the research team identified gaps in the existing content that was found and created new content to fill those gaps. The creation of that content was guided by these same principles.

To inform the development and initial revision of this multimethod adaptive intervention supplement, focus groups with adolescents also will be conducted. 
Focus groups capitalise on group interaction to provide information that would otherwise be unavailable to a researcher. ${ }^{36} 37$ The 30 adolescents who will experience the full L2B programme as part of Aim \#1 will participate in a focus group following the last of the six sessions. Several EMI examples will be presented, and adolescents will be asked to share their reactions to them to facilitate group interaction and to examine what elicits positive and negative responses from adolescents. Discussions will be audio-recorded.

\section{Participants and recruitment}

High school-aged adolescents (14-18 years old) will be recruited for all elements of this process; for all elements of this protocol, parents and adolescents will provide consent and assent, respectively. Recruitment will occur through the Center for Family \& Couple Therapy (CFCT) at Colorado State University, which hosts a mentoring programme and a social group for adolescents. These programmes serve high-risk adolescents who are referred by schools, community agencies and the juvenile justice system of Larimer County; youth are referred if they are at-risk for not reaching their full potential because of significant individual and/or environmental risk. These adolescents are likely to come from families with relatively high levels of conflict. ${ }^{38}$ We will also recruit using advertisements in local school bulletins, community resources and with local therapists not affiliated with the CFCT. Interested adolescents and parents will complete a short screening questionnaire. Adolescents will be eligible if they report (1) occasionally, frequently or very frequently feeling stressed or anxious and (2) that their parents (i) are not divorced or legally separated or pursuing divorce or legal separation and (ii) engage in at least occasional and/or hostile interparental conflict and/or adolescents wish that their parents engaged in less conflict and/or parents report marital distress (scoring $\leq 100$ on the Short Marital Adjustment Test, SMAT).$^{39}$

\section{Aim 2: Refine EMI supplement and determine the best delivery plan}

After collecting and creating content for the EMI supplement, it will then be necessary to determine the most effective means of delivery. To do this, a new group of adolescents will be recruited to experience L2B plus the EMI supplements and opinions will be collected via focus groups, which will aid in refinement of the content to adolescents' liking. This procedure is described next.

\section{Participants and recruitment}

For this aim, 10 high school-aged adolescents (14-18 years old) will be recruited through the CFCT at Colorado State University and local community resources. Screening and eligibility procedures will be the same as detailed above. This sample size was selected to allow us to get feedback on the refinement of the EMI supplement and delivery plan from two cohorts of adolescents (with an estimate of five teens per cohort).

\section{Procedure}

As part of a mixed methods, sequential design, ${ }^{40}$ adolescents will experience L2B Plus and participate in focus groups, which will inform refinement of the multimodal adaptive supplement. Again, in addition to always having access to the on-demand library, adolescents can receive intervention content in two ways: (1) in identified moments of need and (2) randomly throughout the day. First, to identify moments of need, between each group meeting session, adolescents will complete EMA throughout the day (first thing in the morning, $4 \mathrm{pm}$ or after school on weekdays and in the evening) and report feelings of stress, anxiety and experience of family conflict; links to EMA will be texted to teens, who will complete the questions online. When adolescents report elevated stress/anxiety or the experience of family conflict, they will be directed via text message to relevant practices in the on-demand library; this process will happen automatically. More specifically, when teens report elevated symptoms (eg, levels of stress rated as at least a 7 on a 10 point scale), adolescents will automatically be sent text messages with brief intervention content as well as a link to a relevant practice in the on-demand library. Second, at several random intervals throughout the day, adolescents will receive text-message reminders and/or encouragements to engage in mindfulness. Across the 6 -week intervention, the dosage of this EMI (ie, the number of text messages received) will be varied between 1 and 5 times per day; the order of dosage will be counterbalanced across weeks as well as across participants. Quantitative assessments of the acceptability of EMI content will occur using surveys of how much adolescents like the EMI $(0=$ not at all to $5=$ verymuch; N/A: didn't do activity). To evaluate the frequency of EMI that is best-liked by adolescents, each week, adolescents will report how many text messages they would like to receive in relation to the number they received the prior week (more, less or the same number). Using these reports, we will identify the activities, modalities (ie, text, video or audio) and dosages that were both the most and least (1) liked and (2) completed. We will use this quantitative information to inform the focus group discussion, which will occur at the beginning of the subsequent group meeting. In these focus group discussions, the moderator will lead adolescents through the activities that were rated the most and least liked and use a discussion guide to probe for whether and why adolescents did or did not like by these activities as well as why adolescents did and did not complete activities. Responses to different dosages will also be elicited. To facilitate disclosure, adolescents will be instructed that the purpose is to improve the programme and that critical feedback is appreciated. This methodological strategy will allow for evaluation of each individual activity as well as deeper understanding (through complementary interactions of agreement and argumentative interactions of disagreement ${ }^{41}$ of the characteristics that drive adolescent liking of EMI activities. 
Immediately following each week's focus group, that week's L2B session will take place. At the end of the programme, overall reactions about what was most and least liked/helpful will be assessed via questionnaires and individual semistructured interviews.

\section{Data analysis and expected outcomes}

Qualitative data from focus groups will be analysed following an inductive approach. ${ }^{42}$ In addition, repeated measures analysis of variance (ANOVA) will be used to examine whether liking ratings or adherence (ie, proportion of activities that adolescents completed) differ based on modality/dosage. These analyses will inform EMI modifications to maximise adherence and acceptance. Themes for improvement that arise in both qualitative and quantitative analyses will guide EMI refinement. This information will be triangulated with clinical judgments of the research team as well as the literature to guide decisions about how to refine the EMI content.

\section{Aim 3: Examine the feasibility and acceptability of L2B Plus Participants and recruitment}

Participants will be 38 newly recruited adolescents from high-conflict homes and both of their parents, for a total of 114 individuals. Primary recruitment will again occur through the CFCT and community resources.

Adolescents and one parent will complete separate screening measures (online or over the phone). Parents will report the number of adults in the home and adolescent age and complete the SMAT. ${ }^{39}$ Adolescents will report their stress, anxiety and appraisals of their parents conflict as well as complete the Patient Health Questionnaire-9item. ${ }^{43}$ Families will be included in the study if the following criteria are met: (1) there are two parents in the home, who are not pursuing legal separation or divorce and are willing to engage in marital education (2) the adolescent in the home is between 14 and 18 years of age (ie, $>13$ and $<19$ ), (3) adolescents are not clinically depressed and do not report suicidal ideation at baseline, (4) adolescents report that their parents engage in at least occasional and/or hostile interparental conflict and/or conflict that they feel threatened by or self-blame for parents' conflict and/or parents report marital distress (scoring $\leq 100$ on the SMAT) ${ }^{39}$ and (5) the adolescent reports occasionally, frequently or very frequently feeling stressed or anxious.

\section{Procedure}

A preliminary (Phase II) randomised controlled trial will be used to test L2B Plus. All parents will receive 6 weeks of free marital education. Families will be randomly assigned using urn randomisation (balancing groups by adolescent sex and age) to either the L2B Plus condition $(n=19)$ or an active, health education control condition $(n=19)$. The Health Education Wellness Program (modelled after Hey Durham $)^{44}$ will provide didactic information on substance use, nutrition/body image, stress management, exercise and signs of depression/suicide. The choice of control group was motivated by the goal of Aim \#4 (which will be tested using these same data) to compare a mindfulnessgroup to an attention-matched non-mindfulness group. The principal investigator as well as individuals involved in outcome assessments and data entry/analyses, will be blind to participant condition. To maintain the blind, research team members who are not the principal investigator (PI) or involved in outcome assessment or data entry/analyses will implement the randomisation procedure and prepare unblinded reports. Intervention facilitators will not necessarily be blinded to study condition, but will not be involved with outcome assessment or data entry/analyses. The most likely condition in which the blind will be broken is that participants disclose their group assignment to someone on the assessment team. We will engage in training procedures so that assessment team members are well-prepared to handle this breaking of the blind. We will put procedures in place so that such a breaking of the blind would not threaten the integrity of the data. Data will be entered into REDCap either by participants or by team members who are specifically trained in data entry and are not involved in assessment. The blind will be broken only by study team members under critical incident conditions. Research team members responsible for randomisation and preparing unblinded reports as well as intervention facilitators will be authorised to break the blind if one of the participants has a very serious adverse reaction or event. Then, the PI will be informed to help assure that the procedures of the data safety and monitoring procedures are undertaken. There will be no reason that the blind will be broken with other study team members. Also, the PI will not be involved in assessment.

The L2B Plus condition will include the 6 week, in person L2B intervention, supplemented with the textmessaging and online supplement to L2B ('Plus') developed in the first aim. Adolescents will be sent EMI text messages several times a day (with reminders, encouragement and guides to practice mindfulness) and will also have access to the on demand online library of mindfulness resources.

Both group interventions will be administered by graduate students who will receive extensive training in implementation and supervised by the first author (RGLT) as well as programme developer (PB). To be certified, potential facilitators need: (1) a foundation of mindfulness practice (eg, participating in a MBSR course), (2) relevant professional expertise (eg, teaching and/or counselling experience); (3) participation in 24 hours of training by a master training (including a foundational training about the background of L2B, plus an intensive training about facilitation involving demonstrations of L2B lessons and discussions and opportunities to practice leading sessions with feedback from experienced trainers) and (4) individual supervision and coaching (ie, practice leading full L2B sessions with feedback from experienced trainers). In the current study, minimum qualifications for L2B facilitators will be a Bachelor's 
degree and specific and thorough training in programme implementation. In addition, facilitators will be current or former students in Marriage and Family Therapy who have received extensive clinical training and supervision. Each intervention consists of six 90 min sessions delivered weekly for a total of 9 contact hours each. Both programmes will be administered in a classroom at Colorado State University. There will be multiple instructors for each intervention group.

Data will be gathered to evaluate the feasibility and acceptability of each element of L2B Plus (ie, in-person meetings, EMI content, marital education; see table 1).

Table 1 Feasibility and acceptability measures

\begin{tabular}{cl}
\hline & Specific indicator \\
\hline Primary endpoints & \\
Safe implementation & $<10 \%$ will display increased \\
& symptomatology across the intervention
\end{tabular}

\section{Assessment tool}

Daily assessments of perceived stress (Perceived Stress Scale short form ${ }^{57-59}$ ) and anxiety symptoms (State-trait anxiety inventory, Speilberger, ${ }^{58-60} 2010$ ).

\begin{tabular}{|c|c|c|}
\hline Recruitment of target $\mathrm{N}$ & $\begin{array}{l}\text { Successful recruitment of } 38 \text { families (114 } \\
\text { individuals) meeting inclusion criteria }\end{array}$ & Time to recruit target $\mathrm{N}$ \\
\hline Enrolment of \% eligible & \% eligible enrolled & $\begin{array}{l}\text { Study team will track individuals who are } \\
\text { eligible and do/do not enrol }\end{array}$ \\
\hline Retention to intervention & $\begin{array}{l}\geq 90 \% \text { of adolescents will attend at least } \\
4 / 6 \text { sessions }\end{array}$ & $\begin{array}{l}\text { Attendance will be tracked by session } \\
\text { facilitator }\end{array}$ \\
\hline
\end{tabular}

Secondary endpoints

Feasibility indicators

L2B \& control group successful $\quad$ Fidelity $\geq 80 \%$

implementation

Fidelity checklist completed by RAs who will observe each session live for curriculum adherence, group participation, facilitator delivery and facilitation processes.

L2B only: programme developer will evaluate recorded sessions using the TMEOS $^{45}$

\begin{tabular}{|c|c|c|}
\hline (L2B) Plus successful implementation & $\begin{array}{l}<5 \% \text { across sessions of adolescents } \\
\text { reporting that they did not receive EMI } \\
\text { content in response to requests or did not } \\
\text { receive EMI each day }\end{array}$ & Reported by adolescent participants \\
\hline Health and wellness education & $\begin{array}{l}\geq 90 \% \text { of adolescents will attend } 4 / 6 \\
\text { sessions }\end{array}$ & Attendance will be tracked by facilitator \\
\hline \multicolumn{3}{|l|}{ Acceptability indicators } \\
\hline L2B and control group & $\begin{array}{l}\geq 80 \% \text { will rate liking of content as } \geq 4 \\
\text { ( } 1=\text { not at all, } 2=\text { slightly, } 3=\text { moderately, } \\
4=\text { very much, } 5=\text { extremely) }\end{array}$ & Weekly survey for adolescents \\
\hline Health and wellness education & $\begin{array}{l}\geq 80 \% \text { will rate liking/helpfulness as } \geq 4 \\
\text { ( } 1=\text { not at all, } 2=\text { =slightly, } 3=\text { moderately, } \\
4=\text { =very much, } 5=\text { =xtremely) }\end{array}$ & Weekly survey for adolescents \\
\hline
\end{tabular}

EMI, ecological momentary intervention; L2B, Learning to BREATHE; RAs, research assistants; TMEOS, Teaching Mindfulness in Education Observation Scale. 
Feasibility of high-quality implementation for both L2B Plus and control conditions will be evaluated by trained, research assistants who will observe each session live. The L2B programme creator also will observe L2B session video-recordings and evaluate both fidelity and facilitator competence using the Teaching Mindfulness in Education Observation Scale (TMEOS), a reliable and valid observational rating tool for assessing fidelity and quality of implementation. More specifically, the TMEOS assesses proficiency of (1) procedural adherence (ie, planning, organisation and curriculum coverage), (2) teaching mindfulness, (3) guiding mindfulness practices and (4) management of the learning environment. ${ }^{45}$ Both reports of fidelity by the live coders and the programme creator will be reported, and evidence for successful implementation will be considered if ratings of $\geq 80 \%$ fidelity by both live coders and the programme creator are achieved.

\section{Aim 4: Testing L2B Plus and explore the extent to which increasing mindfulness appears to reduce dysregulated stress and anxiety}

Finally, L2B Plus in its refined state will be tested for its ability to reduce stress and anxiety in teens from high conflict homes, relative to an active control condition. This portion of the study is described here. This study is registered with clinicaltrials.gov (ID NCT03869749).

\section{Participants and recruitment}

This aim will be tested with the same participants as described under Aim \#3.

\section{Procedure}

In addition to the procedures described under Aim \#3, baseline and post-test assessments will be conducted through during a family visit and also a group data collection for adolescents in the first and last week of the group meeting (see tables 2 and 3). Baseline assessments will occur within 1 month before the start of the intervention and post-test assessments will occur within 2 weeks following the last group meeting; follow-up assessments will occur 3 months (plus or minor 2 weeks) of the last group meeting. Prior to the follow-up assessment, adolescents will complete questionnaires online and then complete the assessment in-person. Parents will engage in a conflict discussion ${ }^{46}$ which will be video-recorded and later coded for negative and positive conflict behaviours. Adolescents will experience the Trier Social Stress Test (TSST), ${ }^{47}$ a robust, standardised stressor; cardiovascular and cortisol reactivity to and recovery from this stressor will be measured. In addition, adolescents will complete a computerised measure of distress tolerance, a manifestation of a dimension of mindfulness, via the Behavioral Indicator of Resiliency to Distress. ${ }^{48}$ Parents will report on family demographics, adolescent anxiety as well as the frequency, intensity and resolution of marital conflict. Adolescents will report on trait mindfulness, selfcompassion, anxiety and depression, perceived stress and appraisals of interparental conflict. In addition, for 2 days after the laboratory assessment, adolescents will provide saliva samples to assess diurnal cortisol production.

In addition, in between each meeting sessions, participants will complete daily ecological assessments at multiple, random intervals throughout the day and report state mindfulness, frequency of mindfulness practices, state anxiety, state stress and observed or experienced family conflict.

\section{Data analytic considerations}

In line with recommendations for pilot studies, ${ }^{49}$ inferential statistics are not proposed and therefore power analyses are not necessary given that the sample size was selected to allow us to adequately assess feasibility and acceptability. The plan is to (1) descriptively examine change in relevant outcomes in both conditions and (2) conduct inferential statistics to examine within-person links between mindfulness, stress and anxiety, rather than as a test of intervention effects.

In addition to the descriptive statistics outlined above to test primary and secondary hypotheses, exploratory ANOVAs will be estimated to examine group differences at post-test and follow-up. In addition, repeated measures ANOVAs will be used to examine average changes from pretest to post-test and follow-up for each intervention condition. We will use these ANOVAs to construct $95 \%$ CIs around these differences in magnitude to estimate the degree of uncertainty in these estimates. Although underpowered, these analyses will allow us to explore potential group differences that will inform a future adequately powered trial. It is expected that some missing data will be present in the final datasets. We will conduct a thorough missing data analysis and examine whether any time one or demographic characteristics are predictors of study and/or intervention dropout. To account for missing data, multiple imputation (MI) will be used following the guidelines out forth by Little and Rubin ${ }^{50}$ for creating the imputed datasets, conducting the analyses and combining the results. Variables that systematically predict missingness will be included as auxiliary variables in the MI and as control variables in the exploratory ANOVAs. Given the small sample size in this design, we aim to limit these control variables to a very small number. Groups will be conducted with a minimum of two and a maximum of 10 youth. Therefore, exploratory analyses will adjust for cohort effects. Because analyses nested within intervention groups would be grossly underpowered, we will compute an intraclass correlation to examine the non-independence of measures within groups. In addition, we will explore analyses in which we control for intervention group in our analyses.

We will also conduct inferential statistics to examine within-person links between mindfulness, stress and anxiety as well as observed or experienced family conflict. More specifically, we will conduct both mixed-effects regression models to examine the extent to which on days that adolescents observe/experience family conflict they are less mindful and similarly whether less mindful days are also 
Table 2 Assessments for Moving 2 Mindful study protocol

\begin{tabular}{|c|c|c|c|}
\hline Domain & Construct & Measure & Description \\
\hline \multicolumn{4}{|c|}{ Screening measures } \\
\hline \multirow[t]{3}{*}{ Parent reports } & $\begin{array}{l}\text { Number of adults } \\
\text { in the home }\end{array}$ & One item measure & $\begin{array}{l}\text { Eligible if there are two parents in the home and other } \\
\text { inclusion criteria are met }\end{array}$ \\
\hline & Adolescent age & One item measure & $\begin{array}{l}\text { Eligible if adolescent is between } 14 \text { and } 18 \text { (ie, }>13 \text { and } \\
<19 \text { ) and other inclusion criteria are met }\end{array}$ \\
\hline & Marital conflict & Short Marital Adjustment Test ${ }^{39}$ & $\begin{array}{l}\text { Assesses the degree of marital distress; scoring } \leq 100 \\
\text { indicates clinically significant distress and eligibility }\end{array}$ \\
\hline \multirow[t]{3}{*}{$\begin{array}{l}\text { Adolescent } \\
\text { reports }\end{array}$} & Stress & $\begin{array}{l}\text { One item measure; 'How often do you feel } \\
\text { stressed out?' (never, very rarely, rarely, } \\
\text { occasionally, frequently or very frequently) }\end{array}$ & $\begin{array}{l}\text { Youth are eligible if they report occasionally, frequently or } \\
\text { very frequently feeling stressed or anxious }\end{array}$ \\
\hline & Anxiety & $\begin{array}{l}\text { One item measure, 'How often do you } \\
\text { feel anxious?' (never, very rarely, rarely, } \\
\text { occasionally, frequently or very frequently) }\end{array}$ & $\begin{array}{l}\text { Youth are eligible if they report occasionally, frequently or } \\
\text { very frequently feeling stressed or anxious }\end{array}$ \\
\hline & $\begin{array}{l}\text { Reports of } \\
\text { interparental } \\
\text { conflict }\end{array}$ & $\begin{array}{l}\text { Three items: 'How often do your parents } \\
\text { argue?', 'When your parents argue, how } \\
\text { often is it really negative or angry?' and } \\
\text { 'How often are you worried that something } \\
\text { bad will happen when your parents fight } \\
\text { AND/OR feel like it's your fault that your } \\
\text { parents are fighting?' (never, very rarely, } \\
\text { rarely, occasionally, frequently, very } \\
\text { frequently) }\end{array}$ & $\begin{array}{l}\text { Youth are eligible if they answer occasionally, frequently or } \\
\text { very frequently to any of these questions }\end{array}$ \\
\hline
\end{tabular}

Baseline, post-test and follow-up assessment measures

$\begin{array}{lll}\begin{array}{l}\text { Adolescent } \\ \text { reports }\end{array} & \text { Trait mindfulness } & \begin{array}{l}\text { Mindfulness Awareness and Attention Scale, } \\ \text { Adolescent Version }\end{array}\end{array}$

Assesses the extent to which an individual is typically focused on the present moment

Assesses several areas of trait self-compassion, inclum
self-kindness, self-judgement, common humanity, isolation, mindfulness and overidentification

Emotion regulation Difficulties in Emotion Regulation Scale ${ }^{63}$

Assesses multiple dimensions of emotion regulation/ dysregulation, including: non-acceptance of emotional responses, difficulties engaging in goal-directed behaviour impulse control difficulties, lack of emotional awareness, limited access to emotional awareness strategies and lack of emotional clarity

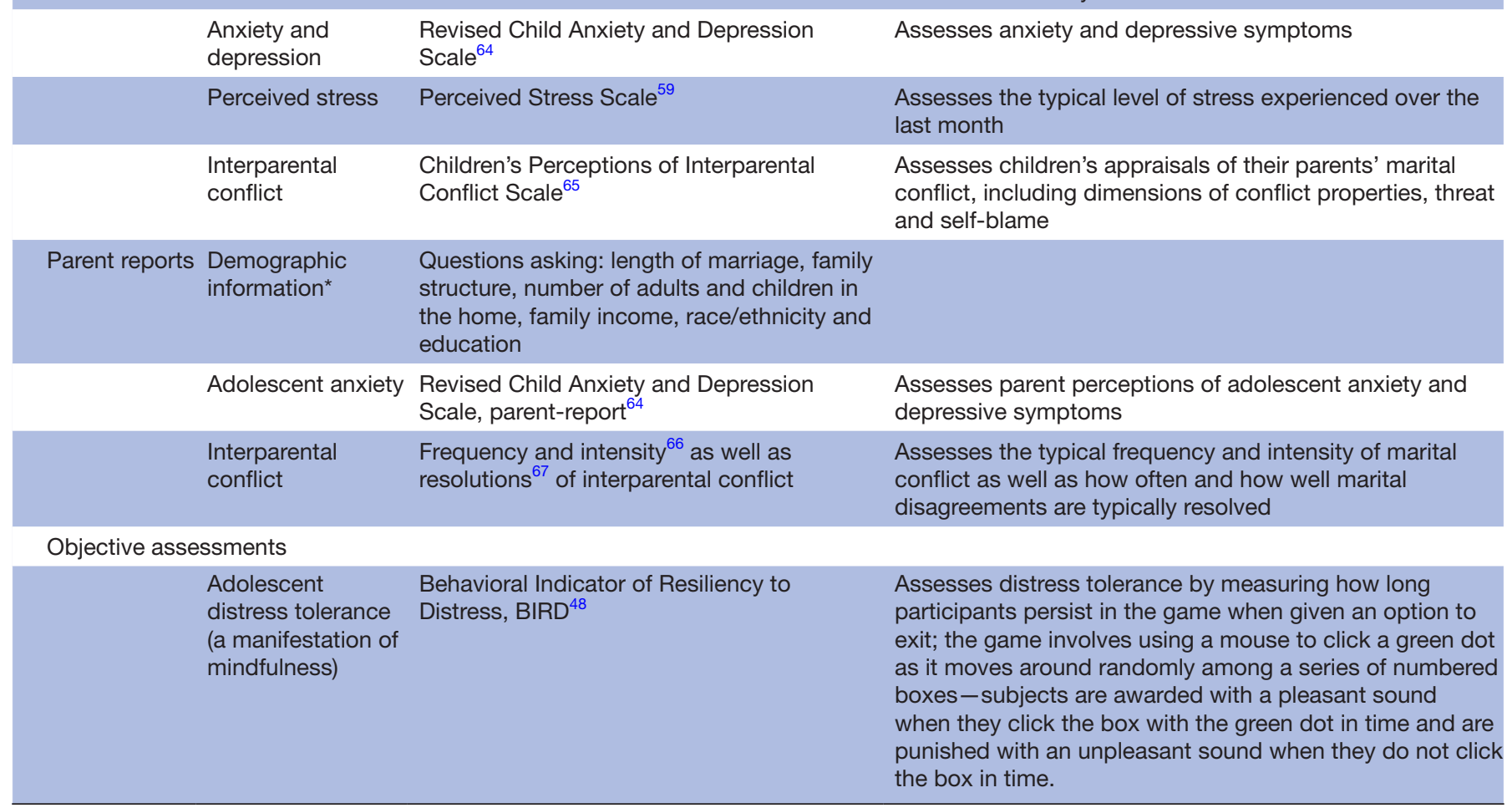


Table 2 Continued

\begin{tabular}{|c|c|c|}
\hline Domain & Construct & Measure \\
\hline & $\begin{array}{l}\text { Adolescent } \\
\text { cardiovascular and } \\
\text { cortisol reactivity } \\
\text { to a standardised } \\
\text { stressor }\end{array}$ & $\begin{array}{l}\text { Reactivity to Trier Social Stress Test }{ }^{47} \text { (from } \\
20 \mathrm{~m} \text { baseline of watching a neutral video) } \\
\text { heart rate, blood pressure: } 3 \text { min intervals } \\
(\triangle \text { from baseline to TSST) } \\
\text { Cortisol: saliva samples after baseline } \\
\text { and immediately as well as } 10 \text { and } 20 \mathrm{~m} \\
\text { after TSST }\end{array}$ \\
\hline & $\begin{array}{l}\text { Cortisol } \\
\text { production } \\
\text { across the day }\end{array}$ & $\begin{array}{l}\text { Diurnal cortisol, from samples taken the } \\
2 \text { days after each visit }\end{array}$ \\
\hline & $\begin{array}{l}\text { Observed } \\
\text { marital conflict } \\
\text { behaviour during } \\
\text { a standardised } \\
\text { conflict } \\
\text { discussion }\end{array}$ & $\begin{array}{l}\text { Partners will engage in a conflict discussion } \\
\text { which will be coded for positive as well as } \\
\text { negative conflict behaviours by objective } \\
\text { observers }\end{array}$ \\
\hline
\end{tabular}

Description

The TSST involves a short speech task and math task. In the speech task, adolescents give a speech to an evaluator who is trained to appear neutral and refrain from giving affirmative responses and adolescents are told their speech will be video recorded and later analysed my experts. In the math task, participants are asked to quickly do mental math while being evaluated in a similar manner.

Participants will be asked to provide samples on awakening, 30 min after waking, at 16:00 hours and before brushing their teeth for bed. These samples will be used to measure cortisol awakening responses as well as cortisol slopes

The conflict discussion will last $10 \mathrm{~min}$; participants will be asked to talk about the largest areas of conflict in their relationship and try to reach an agreement

\begin{tabular}{|c|c|c|c|}
\hline \multicolumn{4}{|c|}{ Daily ecological momentary assessments-answered by adolescents every day of the intervention } \\
\hline \multirow[t]{4}{*}{$\begin{array}{l}\text { Adolescent } \\
\text { reports }\end{array}$} & $\begin{array}{l}\text { State } \\
\text { mindfulness }\end{array}$ & $\begin{array}{l}\text { Mindful Attention and Awareness Scale, } \\
\text { State }^{68}\end{array}$ & Assesses the days' level of present-moment awareness \\
\hline & $\begin{array}{l}\text { State perceived } \\
\text { stress }\end{array}$ & Perceived Stress Scale, State ${ }^{59}$ & Assesses the days' level of stress \\
\hline & $\begin{array}{l}\text { State anxiety \& } \\
\text { depression }\end{array}$ & Three items, on a scale from 1 to 10 & $\begin{array}{l}\text { Asks participants to report how often they felt (1) on } \\
\text { edge, unable to concentrate, nervous, uneasy, (2) sad, } \\
\text { hopeless, discouraged, (3) exhausted, worn out }\end{array}$ \\
\hline & Self-compassion & Two items, on a scale from 1 to 10 & $\begin{array}{l}\text { Asks participants to report the extent to which they were } \\
\text { (1) understanding and patient with themselves and (2) } \\
\text { disapproving and judgmental of their own flaws }\end{array}$ \\
\hline & $\begin{array}{l}\text { Mindfulness } \\
\text { practices } \\
\text { completed since } \\
\text { the last session }\end{array}$ & One item & $\begin{array}{l}\text { Asks participants to report how many mindfulness } \\
\text { practices they completed since the last assessment }\end{array}$ \\
\hline & EMI receipt & Two items & $\begin{array}{l}\text { Asks participants to report whether they received text } \\
\text { messages or used the on-demand library }\end{array}$ \\
\hline
\end{tabular}

*Baseline, post-test and follow-up measures are identical except for the fact that demographic measures will only be collected at baseline. EMI, ecological momentary intervention; TSST, Trier Social Stress Test.

days in which adolescents report greater stress and anxiety. Given the richness of the longitudinal data (ie, EMA collected at least once per day across the 8-week intervention period), we will also conduct heterogeneous mixedeffects models, ${ }^{51}$ which model not just random intercepts (as with mixed-effects regression models) but also random variances, allowing us to model and predict varying erraticism within people (eg, are adolescents more variable in their mindfulness on days that they observe/experience family conflict). These analyses will focus on within rather than between person differences, given the small sample size. We will also model whether mindfulness becomes less erratic over the course of the intervention, as adolescents develop a personal mindfulness practice.

Based on past research using $\mathrm{L} 2 \mathrm{~B},{ }^{25}$ we estimate $15 \%$ attrition. To minimise missing data, we will implement several strategies. Families will enter all questionnaire data directly into REDCap, ${ }^{52}$ in which study team members will identify and review missing data. If missingness exceeds $10 \%$, we will contact participants to encourage them to provide the missing data. This missing review will be conducted with 2 weeks of each assessment. Following an intent-to-treat approach, once a family is randomised, they will always be included in analyses, even if they withdraw 


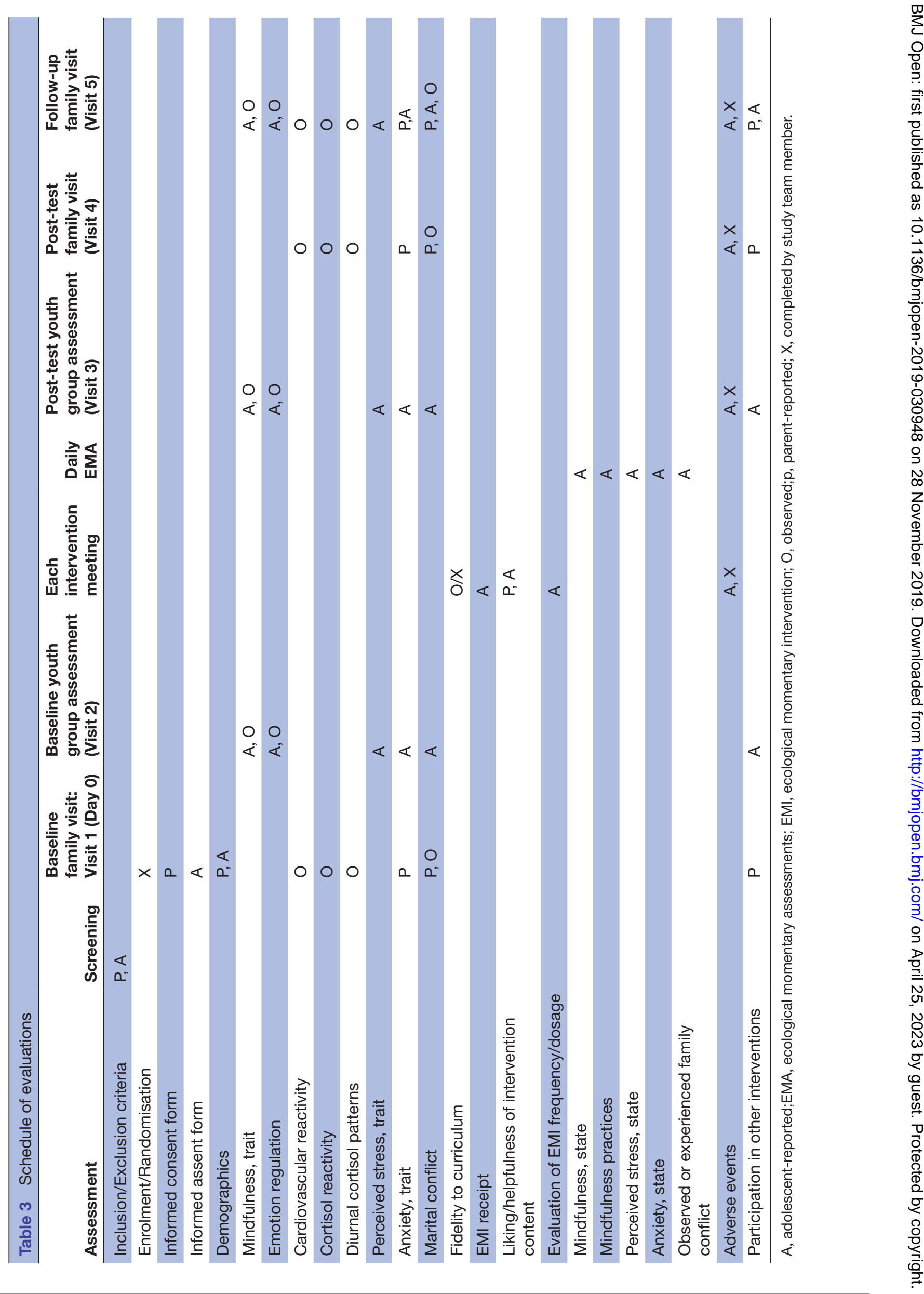

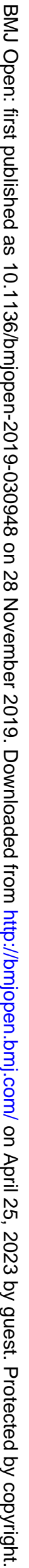


from the intervention or the study or if they access the other treatment. If there is a pool of eligible families who have not yet been randomly assigned, we will randomly select one family to take the place of early withdrawals. Follow-up analyses of the per protocol population will omit any cases for which there were assignment protocol violations (of which we expect very few).

\section{ETHICS AND DISSEMINATION}

This study is approved by the Institutional Review Board at Colorado State University and will follow all regulations designed to protect human participants in research. The results of each stage of this proposed study will be disseminated at scientific conferences and in scientific journals, whether the results are positive, negative or inconclusive.

\section{Limitations}

This study will provide unique and important information about the extent to which directly intervening with adolescents from high-conflict homes to promote mindfulness is a feasible, acceptable and potentially effective strategy to reduce stress and anxiety. However, a multimodal adaptive intervention supplement to L2B might also be applied more broadly to promote mindfulness among adolescents. One of the main strengths of the design is the mixed method longitudinal assessment. Limitations of this study include its small sample size, which limit the generalisability of findings and reduces statistical power. In addition, a potential challenge of the current measurement strategy is habituation with repeated exposure to the TSST. Reduced responding to the TSST does occur, ${ }^{53-57}$ but reactivity is displayed even with exposures on backto-back days. ${ }^{5457}$ As participants will be exposed to the TSST several months apart, it should produce robust physiological stress responses at each testing. It is possible that L2B Plus will not produce change compared with the control condition. We will also conduct exploratory moderator analyses; specifically, we will examine theoretically and empirically supported moderators of effects, including whether L2B Plus appeared beneficial for adolescents in the absence of change in parents' marital relationship and whether change varied based on preintervention symptom severity or amount of daily mindfulness practice. We also would examine the control group to identify elements to modify to maintain attentionmatching without improving outcomes.

\section{CONCLUSION}

We believe that this study is innovative, unique and important. The proposed study is significant because it will provide critical new information about the efficacy of directly targeting adolescents from high-conflict homes with an innovative mindfulness-based intervention to decrease stress and anxiety. This study will develop an intervention model for adolescents from high-conflict homes, but we believe this intervention model may eventually be applied more broadly to adolescents dealing with chronic environmental stressors. To support this effort, the current study will fill gaps in knowledge about within-person links between mindfulness, stress and anxiety. This study is innovative, as it seeks to shift the emphasis from intervening solely to improve marital relationships to also improving adolescent regulatory capabilities. This represents a test of a unique theoretical question (does a mindfulness intervention improve adolescent functioning in the context of marital conflict?) as well as employing a novel approach (incorporating a multimethod adaptive intervention into a mindfulness intervention). At the completion of this study, we expect to have generated evidence for a feasible and acceptable mindfulness plus multimethod adaptive programme that likely produces meaningful reductions in stress and anxiety. We plan to build on the results by preparing a larger-scale efficacy trial of L2B Plus for improving outcomes in adolescents from highconflict homes.

\section{Author affiliations}

${ }^{1}$ Human Development and Family Studies, Colorado State University College of Health and Human Sciences, Fort Collins, Colorado, USA

${ }^{2}$ Psychology, Colorado State University, Fort Collins, Colorado, USA

${ }^{3}$ Biobehavioral Health \& Medicine, Pennsylvania State University, University Park, Pennsylvania, USA

Contributors All authors were involved with study design. RLT and NS wrote the initial draft of the protocol. PCB, JDC, KLH, CJM and JMS read and revised the protocol. All authors approve the publication of this manuscript. A data safety and monitoring committee will be designated, which will review progress reports, including patient recruitment, retention/attrition and adverse experiences. This committee is independent from the sponsor. Protocol amendments will be reviewed by this committee as well as the sponsor and the Institutional Review Board at Colorado State University.

Funding This work was supported by the National Center for Complementary and Integrative Health (Award Number K01AT009592-01; PI, Lucas-Thompson). Publication of this article was supported by the Colorado State University Libraries Open Access Research and Scholarship Fund.

Disclaimer All study documents and information will be stored securely and identified with an identification number to protect participant confidentiality. Participants' study-specific information will not be released to those outside the study team without written permission from the participant.

Competing interests None declared.

Patient consent for publication Not required.

Ethics approval Colorado State University.

Provenance and peer review Not commissioned; externally peer reviewed.

Open access This is an open access article distributed in accordance with the Creative Commons Attribution Non Commercial (CC BY-NC 4.0) license, which permits others to distribute, remix, adapt, build upon this work non-commercially, and license their derivative works on different terms, provided the original work is properly cited, appropriate credit is given, any changes made indicated, and the use is non-commercial. See: http://creativecommons.org/licenses/by-nc/4.0/.

\section{ORCID iD}

Rachel Lucas-Thompson http://orcid.org/0000-0001-7558-2285

\section{REFERENCES}

1 Andersen SL. Trajectories of brain development: point of vulnerability or window of opportunity? Neurosci Biobehav Rev 2003;27:3-18.

2 Ablow JC, Measelle JR, Cowan PA, et al. Linking marital conflict and children's adjustment: the role of young children's perceptions. $J$ Fam Psychol 2009;23:485-99. 
3 Dadds MR, Powell MB. The relationship of interparental conflict and global marital adjustment to aggression, anxiety, and immaturity in aggressive and nonclinic children. J Abnorm Child Psychol 1991;19:553-67.

4 Lucas-Thompson RG. Associations of marital conflict with emotional and physiological stress: evidence for different patterns of dysregulation. J Res Adolesc 2012;22:704-21.

5 Lucas-Thompson RG, Lunkenheimer ES, Dumitrache A. Associations between marital conflict and adolescent conflict appraisals, stress physiology, and mental health. J Clin Child Adolesc Psychol 2017;46:379-93.

6 Lucas-Thompson RG, Lunkenheimer ES, Granger DA. Adolescent conflict appraisals moderate the link between marital conflict and physiological stress reactivity. J Res Adolesc 2017;27:173-88.

7 Turner HA, Kopiec K. Exposure to interparental conflict and psychological disorder among young adults. $J$ Fam Issues 2006;27:131-58

8 Zimet DM, Jacob T. Influences of marital conflict on child adjustment: review of theory and research. Clin Child Fam Psychol Rev 2001:4:319-35.

9 Johnson LE, Greenberg MT. Parenting and early adolescent internalizing: the importance of Teasing apart anxiety and depressive symptoms. J Early Adolesc 2013;33:201-26.

10 Last CG, Hansen C, Franco N. Anxious children in adulthood: a prospective study of adjustment. J Am Acad Child Adolesc Psychiatry 1997;36:645-52.

11 McCoy K, Cummings EM, Davies PT. Constructive and destructive marital conflict, emotional security and children's prosocial behavior. $J$ Child Psychol Psychiatry 2009;50:270-9.

12 National Institutes of Health. Research on children exposed to violence. Research program announcement PAR-03-096, 2003.

13 Faircloth WB, Cummings EM. Evaluating a parent education program for preventing the negative effects of marital conflict. J Appl Dev Psychol 2008;29:141-56.

14 Faircloth WB, Schermerhorn AC, Mitchell PM, et al. Testing the longterm efficacy of a prevention program for improving marital conflict in community families. J Appl Dev Psychol 2011;32:189-97.

15 Blanchard VL, Hawkins AJ, Baldwin SA, et al. Investigating the effects of marriage and relationship education on couples communication skills: a meta-analytic study. J Fam Psychol 2009;23:203-14.

16 Cowan PA, Cowan CP. Controversies in couple relationship education (CRE): overlooked evidence and implications for research and policy. Psychology Public Policy and Law 2014;20:361-83.

17 Zoogman S, Goldberg SB, Hoyt WT, et al. Mindfulness interventions with youth: a meta-analysis. Mindfulness 2015;6:290-302.

18 Biegel GM, Brown KW, Shapiro SL, et al. Mindfulness-based stress reduction for the treatment of adolescent psychiatric outpatients: a randomized clinical trial. J Consult Clin Psychol 2009;77:855-66.

19 Bluth K, Campo RA, Pruteanu-Malinici S, et al. A school-based mindfulness pilot study for ethnically diverse at-risk adolescents. Mindfulness 2016;7:90-104.

20 Broderick PC, Metz S. Learning to breathe: a pilot trial of a mindfulness curriculum for adolescents. Adv Sch Ment Health Promot 2009;2:35-46.

21 Dvořáková K, Kishida M, Li J, et al. Promoting healthy transition to College through mindfulness training with first-year college students: pilot randomized controlled trial. J Am Coll Health 2017;65:259-67.

22 Eva AL, Thayer NM. Learning to BREATHE: a pilot study of a mindfulness-based intervention to support marginalized youth. J Evid Based Complementary Altern Med 2017;22:580-91.

23 Fung J, Guo S, Jin J, et al. A pilot randomized trial evaluating a school-based mindfulness intervention for ethnic minority youth. Mindfulness 2016;7:819-28.

24 Fung J, Kim JJ, Jin J, et al. A randomized trial evaluating schoolbased mindfulness intervention for ethnic minority youth: exploring mediators and moderators of intervention effects. $J$ Abnorm Child Psychol 2019;47:1-19.

25 Metz SM, Frank JL, Reibel D, et al. The effectiveness of the learning to breathe program on adolescent emotion regulation. Res Hum Dev 2013;10:252-72.

26 Shomaker LB, Bruggink S, Pivarunas B, et al. Pilot randomized controlled trial of a mindfulness-based group intervention in adolescent girls at risk for type 2 diabetes with depressive symptoms. Complement Ther Med 2017:32:66-74.

27 Lucas-Thompson RG, Seiter NS, Broderick PC, et al. Mindfulness interventions to reduce impact of interparental conflict on adolescents. J Child Fam Stud 2019;23.

28 Heron KE, Smyth JM. Ecological momentary interventions: incorporating mobile technology into psychosocial and health behaviour treatments. Br J Health Psychol 2010;15:1-39.
29 Heron KE, Everhart RS, McHale SM, et al. Using mobile-technologybased ecological Momentary assessment (EMA) methods with youth: a systematic review and recommendations. $J$ Pediatr Psychol 2017:42:1087-107.

30 Smyth JM, Heron KE. Is providing mobile interventions "just-in-time" helpful? An experimental proof of concept study of just-in-time intervention for stress management. leee Wireless Health 2016:89-95.

31 Versluis A, Verkuil B, Spinhoven P, et al. Changing mental health and positive psychological well-being using ecological momentary interventions: a systematic review and meta-analysis. J Med Internet Res 2016;18:e152.

32 Anderson M, Jiang J. Teens, social media \& technology 2018, 2018. Available: http://www.pewinternet.org/2018/05/31/teens-socialmedia-technology-2018

33 Lenhart A. Teens, smartphones \& texting. Pew Internet \& American Life Project 2012;21:1-34.

34 Lister-Landman KM, Domoff SE, Dubow EF. The role of compulsive texting in adolescents' academic functioning. Psychol Pop Media Cult 2017;6:311-25.

35 Lucas-Thompson RG, Broderick PC, Coatsworth JD, et al. New avenues for promoting mindfulness in adolescence using mHealth. $J$ Child Fam Stud 2019;28:131-9.

36 Peek L, Fothergill A. Using focus groups: lessons from studying daycare centers, 9/11, and Hurricane Katrina. Qualitative Research 2009;9:31-59.

37 Morgan DL. Focus groups. Annu Rev Sociol 1996;22:129-52.

38 Hawkins JD. Predictors of youth violence. Washington, DC: U.S. Department of Justice, 2000.

39 Locke HJ, Wallace KM. Short marital-adjustment and prediction tests: Their reliability and validity. Marriage Fam Living 1959;21:251-5.

40 Small ML. How to conduct a mixed methods study: recent trends in a rapidly growing literature. Annu Rev Sociol 2011;37:57-86.

41 Kitzinger J. The methodology of focus groups: the importance of interaction between research participants. Sociol Health IIIn 1994;16:103-21.

42 Thomas DR. A general inductive approach for analyzing qualitative evaluation data. Am J Eval 2006;27:237-46.

43 Kroenke K, Spitzer RL, Williams JB. The PHQ-9: validity of a brief depression severity measure. J Gen Intern Med 2001;16:606-13.

44 Bravender T. Health, education, and youth in Durham: HEY-Durham curricular quide. 2nd ed. Durham: Duke University, 2005.

45 Broderick PC, Frank JL, Berrena E, et al. Evaluating the quality of mindfulness instruction delivered in school settings: development and validation of a teacher quality observational rating scale. Mindfulness 2019;10:36-45.

46 Lucas-Thompson RG, George MW, Quinn-Sparks AR. Trust: an innovative tool for investigating marital conflict in response to a nove stressor. J Fam Psychol 2016;30:625-32.

47 Kirschbaum C, Pirke KM, Hellhammer DH. The 'Trier social stress test'--a tool for investigating psychobiological stress responses in a laboratory setting. Neuropsychobiology 1993;28:76-81.

48 Lejeuz CWet al. The behavioral indicator of resiliency to distress (BIRD). Unpublished manual 2006.

49 Health, N.C.f.C.a.I. Pilot studies: common uses and misuses, 2017. Available: https://nccih.nih.gov/grants/whatnccihfunds/pilot_studies

50 Little RJ, Rubin DB. Statistical analysis with missing data. 2nd edn. New York: Wiley, 2002

51 Hedeker D, Mermelstein RJ, Berbaum ML, et al. Modeling mood variation associated with smoking: an application of a heterogeneous mixed-effects model for analysis of ecological momentary assessment (EMA) data. Addiction 2009;104:297-307.

52 Harris PA, Taylor R, Thielke R, et al. Research electronic data capture (REDCap)--a metadata-driven methodology and workflow process for providing translational research informatics support. J Biomed Inform 2009;42:377-81.

53 Wüst S, Federenko IS, van Rossum EFC, et al. Habituation of cortisol responses to repeated psychosocial stress-further characterization and impact of genetic factors. Psychoneuroendocrinology 2005;30:199-211.

54 Kirschbaum C, Prüssner JC, Stone AA, et al. Persistent high cortisol responses to repeated psychological stress in a subpopulation of healthy men. Psychosom Med 1995;57:468-74.

55 Kudielka BM, von Känel R, Preckel D, et al. Exhaustion is associated with reduced habituation of free cortisol responses to repeated acute psychosocial stress. Biol Psychol 2006;72:147-53.

56 Schommer NC, Hellhammer DH, Kirschbaum C. Dissociation between reactivity of the hypothalamus-pituitary-adrenal axis and the sympathetic-adrenal-medullary system to repeated psychosocial stress. Psychosom Med 2003;65:450-60.

57 Pruessner JC, Gaab J, Hellhammer DH, et al. Increasing correlations between personality traits and cortisol stress responses obtained by data aggregation. Psychoneuroendocrinology 1997;22:615-25. 
58 Federenko IS, Nagamine M, Hellhammer DH, et al. The heritability of hypothalamus pituitary adrenal axis responses to psychosocial stress is context dependent. J Clin Endocrinol Metab 2004;89:6244-50.

59 Cohen S, Kamarck T, Mermelstein R. A global measure of perceived stress. J Health Soc Behav 1983;24:385-96.

60 Spielberger CD. State-trait anxiety inventory (STAI). Palo Alto: Consulting Psychologists Press, 2010.

61 Brown KW, West AM, Loverich TM, et al. Assessing adolescent mindfulness: validation of an adapted mindful attention awareness scale in adolescent normative and psychiatric populations. Psychol Assess 2011;23:1023-33.

62 Raes F, Pommier E, Neff KD, et al. Construction and factorial validation of a short form of the Self-Compassion scale. Clin Psychol Psychother 2011;18:250-5.

63 Gratz KL, Roemer L. Multidimensional assessment of emotion regulation and dysregulation: development, factor structure, and initial validation of the difficulties in emotion regulation scale. $J$ Psychopathol Behav Assess 2004;26:41-54.
64 Chorpita BF, Yim L, Moffitt C, et al. Assessment of symptoms of DSM-IV anxiety and depression in children: a revised child anxiety and depression scale. Behav Res Ther 2000;38:835-55.

65 Grych JH, Seid M, Fincham FD. Assessing marital conflict from the child's perspective: the children's perception of interparental conflict scale. Child Dev 1992;63:558-72.

66 Braiker H, Kelley H. Conflict in the development of close relationships. In: Burgess R, Huston T, eds. Social exchange and developing relationships. San Diego, CA: Academic Press, 1979: 135-68.

67 Kerig PK. Assessing the links between interparental conflict and child adjustment: the conflicts and problem-solving scales. J Fam Psychol 1996;10:454-73.

68 Brown KW, Ryan RM. The benefits of being present: mindfulness and its role in psychological well-being. J Pers Soc Psychol 2003;84:822-48. 